J. Phys. IV France 127 (2005) 99-103

(C) EDP Sciences, Les Ulis

DOI: $10.1051 /$ jp4:2005127015

\title{
Optimisation de la génération d' harmoniques d'ordre élevé à l'aide d'une optique adaptative et d'un modulateur acousto-optique
}

\author{
O. Boyko ${ }^{1}$, D. Reitze ${ }^{2}$, S. Kazamias ${ }^{3}$, T.A. Planchon ${ }^{1}$, C. Valentin ${ }^{1}$ \\ et $\mathrm{Ph}$. Balcou ${ }^{1}$ \\ ${ }^{1}$ Laboratoire d'Optique Appliquée, ENSTA - École Polytechnique, CNRS, \\ 91761 Palaiseau Cedex, France \\ 2 Physics Department, University of Florida, Gainesville, FL 32611, USA \\ ${ }^{3}$ LIXAM, Université Paris-Sud CNRS, 9405 Orsay, France
}

\begin{abstract}
Résumé. Afin de contrôler la génération d'harmoniques d'ordre élevé dans des conditions optimisées, nous avons réalisé des expériences préliminaires de contrôle spectral et spatial du laser infrarouge. Nous avons démontré la possibilité de modifier la longueur d'onde des harmoniques grâce à la modification de la phase spectrale du laser infrarouge par un modulateur acousto-optique «Dazzler ». Nous montrons que cette méthode permet d'obtenir un spectre plus large et un flux de photon intégré beaucoup plus important qu'en utilisant une simple modification de la dérive de fréquence du laser. Nous présentons également les premiers résultats de mise en forme de la tache focale du laser par optique adaptative, et l'effet de ce contrôle spatial sur la génération d'harmoniques.
\end{abstract}

\section{INTRODUCTION}

La génération d'harmoniques d'ordre élevé est devenue une technique standard pour produire de la lumière cohérente dans le domaine de longueur d'onde d'extrême ultraviolet $(10-50 \mathrm{~nm})$. Ce type de rayonnement est très étudié depuis plus de 10 ans car il permet d'obtenir des faisceaux de qualité laser dans une gamme spectrale que les lasers standards ne peuvent pas couvrir en régime femtoseconde [1]. Grâce aux développements technologiques sur les lasers, il devient aujourd'hui possible d'effectuer un réel " contrôle cohérent" du processus, en particulier en jouant sur les phases spectrales et spatiales du laser de pompe.

En tant que source ultracourte de photons XUV utilisée pour les applications, les harmoniques présentent l'avantage de couvrir une large bande spectrale, à travers les différents ordres impairs harmoniques du plateau. Il est, donc, particulièrement utile de démontrer une accordabilité complète des harmoniques d'ordre élevé sur tout le domaine XUV, comme le peuvent les installations synchrotron. Premièrement, nous décrivons les résultats de contrôle spectral des harmoniques par un modulateur acousto-optique agissant sur la phase spectrale du laser de pompe (Dazzler) [2]. Deuxièmement, nous présentons aussi une nouvelle méthode de mise en forme de taches focales du laser et en particulier, la création d'un minimum local d'intensité au foyer du laser. Par le contrôle de la phase spatiale du laser, il est possible d'obtenir un profil «creusé » de la distribution d'intensité au foyer. Lors d'expériences d'interaction entre un laser ultra-haute intensité et un plasma, cette méthode permet par exemple de piéger les électrons dans la zone focale, grâce au rôle de la force pondéromotrice. La génération des harmoniques élevées peut bénéficier aussi de cette méthode. Par le contrôle du profil radial de l'intensité I(r) au foyer du laser, nous modifions le régime d'accord de phase pour les harmoniques et, par conséquent, les caractéristiques des harmoniques et la durée d'émission. Nous montrons ainsi les effets de modification de la phase spatiale du laser sur la génération d' harmoniques d'ordre élevé. 


\section{CONTRÔLE SPECTRAL ACTIF DES HARMONIQUES}

Nous avons utilisé un laser Titane :Saphir de longueur d'onde centrale de $810 \mathrm{~nm}$. Le spectre produit dans l'UVX se présente sous la forme d'un peigne de plusieurs harmoniques d'ordre élevé, dont les pics d'énergies sont séparées de $3 \mathrm{eV}$. Afin de contrôler spectralement les harmoniques et idéalement de pouvoir déplacer une harmonique donnée de $1.5 \mathrm{eV}$ pour obtenir une accordabilité totale des harmoniques, nous avons utilisé un modulateur acousto-optique dispersif programmable (AOPDF), connu sous le nom de «Dazzler », qui permet simplement et efficacement de modifier la phase spectrale du laser. Ce dispositif est généralement utilisé pour contrôler la phase spectrale et l'amplitude spectrale dans les chaînes lasers $[3,4]$.

Des premiers essais de contrôle spectral des harmoniques d'ordre élevé faisaient appel à l'introduction d'une simple dérive de fréquence, liée à un dérèglement contrôlé du compresseur d'impulsions. En principe la dérive de fréquence linéaire ainsi introduite change la fréquence instantanée au moment de l'émission sur le front montant de l'impulsion laser et par conséquent, décale le spectre d'harmoniques [5]. Cette méthode permet d'accorder en fréquence les harmoniques dans une gamme de longueurs d'ondes très courtes $(<20 \mathrm{~nm})$, mais au-dessus de cette valeur présente des limitations marquées : l'accordabilité n'est plus que très partielle, et le nombre de photons produits diminue significativement pour les dérives de fréquence importantes. Dans notre expérience, nous avons utilisé le Dazzler pour modifier la phase spectrale du laser de manière plus complexe par le biais d'une méthode adaptative plus avantageuse car elle permet de produire beaucoup plus de photons dans la gamme de 20-40 nm.

Le laser de cadence $1 \mathrm{kHz}$, d'énergie $6 \mathrm{~mJ}$, de durée d'impulsion de 35 fs est focalisé par une lentille de 1 mètre de focale. La cellule remplie d'argon est placée à $2 \mathrm{~mm}$ après le foyer. La pression du gaz y est de 15 torr, l'ouverture du diaphragme placé avant la lentille est $11 \mathrm{~mm}$. L'intensité au foyer était alors de $3 \times 10^{14} \mathrm{~W} / \mathrm{cm}^{2}$. Nous avons montré que l'optimisation des harmoniques est obtenue en géométrie de focalisation douce (longueur de Rayleigh très importante ( $>17 \mathrm{~mm}$ )) [6]. Les harmoniques produites sont ensuite analysées par un spectrographe XUV. Le Dazzler a été disposé avant le premier étage d'amplification dans la chaîne laser. Nous avons développé un algorithme génétique qui assure l'asservissement entre le Dazzler et la camera CCD du spectromètre. La phase spectrale choisie par l'algorithme génétique est appliquée au Dazzler via une paramétrisation à 10 degrés de liberté : les premiers coefficients $\phi_{2}$ et $\phi_{3}$ du développement de la phase en série de Taylor, ainsi que 8 valeurs de phase choisies entre 0 et $2 \pi$ sur la gamme spectrale couverte. Chaque spectre enregistré par la caméra CCD a été analysé de la manière suivante : les intensités des harmoniques ont été ajustées par des Gaussiennes ; ensuite le nombre de photons compris dans tout le spectre d'une part, la finesse et l'amplitude spectrale d'une harmoniques donnée d'autre part, et enfin sa longueur d'onde centrale ont été comparés avec des valeurs cibles définies au départ. Une fonction de coût quantifie la différence entre le spectre réel et le spectre souhaité, permettant à l'algorithme génétique de tester la qualité du spectre obtenu pour une série de « générations » successives, et de converger (ou non) vers une phase spectrale donnant un spectre optimal.

La figure 1 montre un exemple d'optimisation de l'harmonique 27, obtenu grâce à l'algorithme génétique. Nominalement centrée à $30 \mathrm{~nm}$, elle a été déplacée à $31,16 \mathrm{~nm}$ en cours d'exécution de l'algorithme génétique. L'efficacité de conversion de rayonnement laser en rayonnement XUV reste proche de $1.4 \times 10^{-6}$. On voit sur la figure les résultats de trois générations : la $1^{\text {ére }}$, la $7^{\text {eme }}$ et la $19^{\text {eme }}$.

La Figure 1.b) donne la phase (échelle d'ordonnées droite) et le profil temporel (échelle d'ordonnées gauche) de l'impulsion laser qui correspond à la meilleure optimisation. Le profil temporel est de manière évidente non quadratique. L'algorithme génétique a donc accordé et optimisé les harmoniques par un processus complexe.

De la même façon nous avons montré que nous étions capables de déplacer l'harmonique 27 générée dans l'argon jusqu'aux énergies des harmoniques 26 et 28 (28.92-31.15nm), permettant un raccord avec les harmoniques voisines 25 et 29. Cela correspond à une accordabilité presque totale autour de l'harmonique 27 [7]. 


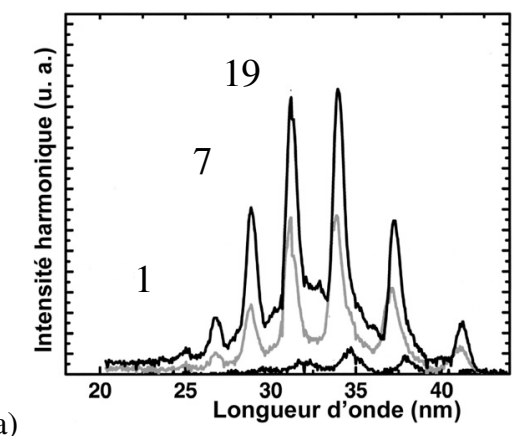

b)

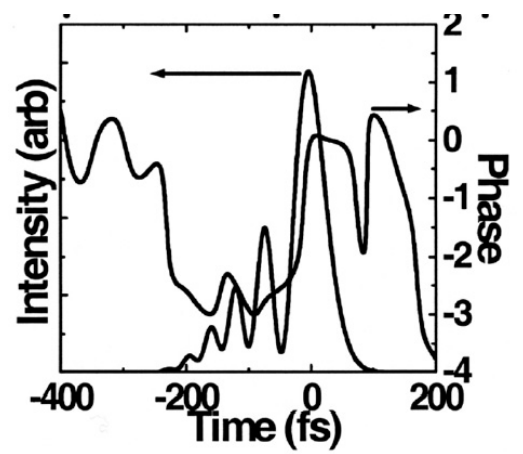

Figure 1. a) L'évolution du spectre harmonique au cours de l'optimisation par l'algorithme génétique. b) Le profil temporel et la phase temporelle de l'impulsion laser correspondant à meilleur spectre harmonique.

\section{CONTRÔLE SPATIAL DU LASER INFRAROUGE}

Une deuxième approche de contrôle du faisceau laser consiste à mettre en forme la répartition spatiale d'intensité du faisceau au foyer. Différentes méthodes de mise en forme des modes spatiaux des lasers ont été proposées, en particulier par des filtres holographiques. Une particularité majeure des faisceaux intenses est d'induire des dommages importants sur la plupart des composants optiques en transmission, si bien que seul les miroirs peuvent être utilisés. La seule méthode envisageable consiste donc en l'utilisation de miroirs déformables, aptes à résister au flux du laser.

Dans le cas précis de l'obtention d'un profil d'intensité au foyer présentant un minimum local, un groupe du LLE de Rochester [8] a proposé d'imposer un déphasage de $\pi$ sur un cercle central du faisceau laser avant focalisation (Figure 2); il existe un diamètre optimal de cette couronne pour lequel des interférences destructives interviennent au foyer entre zone centrale et zone latérale du faisceau initial, créant ainsi un minimum d'intensité au centre de la tache focale.

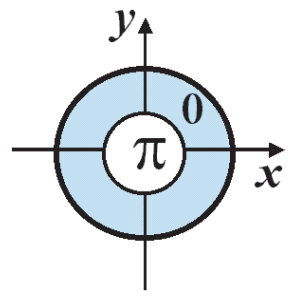

Figure 2. Modification de la phase spatiale du laser avant sa focalisation.

En pratique, Chaloupka et al. ont utilisé une lame demi-onde, dont la partie centrale a été découpée, puis repositionnée en superposant axe rapide central/axe lent latéral ; la lame demi-onde est alors orientée avec cet axe suivant la polarisation du laser. Ce dispositif très ingénieux a permis de montrer l'effet piège des minima d'intensité sur les électrons relativistes; il n'est néanmoins pas généralisable aux lasers ultra-intenses ultra-courts, et ne se prête pas à des expériences de contrôle actif.

Nous avons choisi l'optique adaptative pour le contrôle actif de l'intensité et de la phase spatiale du laser infrarouge. Pour réaliser une correction active du front d'onde, deux éléments sont nécessaires: un analyseur de front d'onde pour mesurer les défauts de phase spatiale et une optique active pour les corriger. Ces deux éléments peuvent fonctionner en boucle fermée, la mesure de front d'onde étant envoyée à l'optique active après passage par un algorithme de correction. Le dispositif expérimental utilisé est détaillé sur la figure 3. Le laser est envoyé sur le miroir déformable puis focalisé par une lentille de $1 \mathrm{~m}$ 


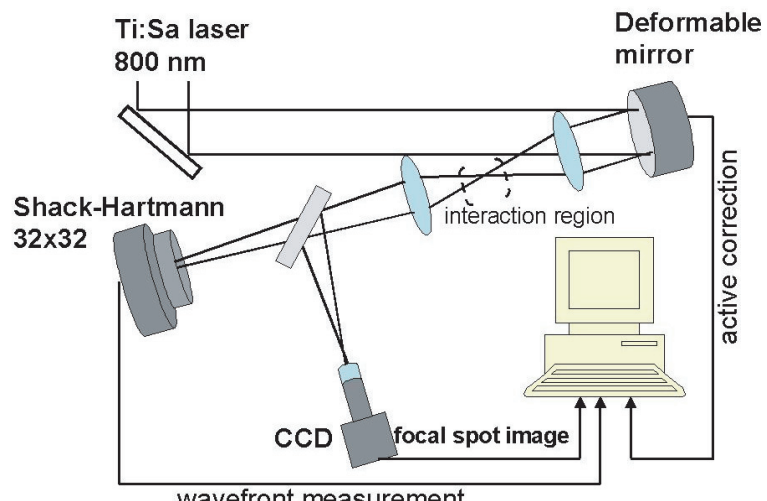

wavefront measurement

Figure 3. Montage expérimental.

de focale (région d'interaction). Le faisceau réfléchi sur le miroir déformable est imagé au moyen d'un doublet sur un senseur de front d'onde de type Shack-Hartmann. La lame séparatrice sépare le faisceau en deux avant le senseur de Shack-Hartmann, permettant d'analyser simultanément le front d'onde du faisceau au niveau du miroir déformable grâce au système d'imagerie et d'observer en parallèle la tache focale sur la caméra CCD. Ainsi, nous contrôlons chaque modification du front d'onde avant focalisation et observons les effets sur la focalisation avec la caméra CCD.

Le miroir déformable utilisé dans cette expérience est un miroir bi-morphe, c'est à dire dont les actionneurs piézoélectriques changent leurs courbures en fonction des tensions appliquées. Le miroir est couplé avec le senseur et piloté par ordinateur. L'utilisation du miroir déformable pour créer le saut de phase de $\pi$ pour la partie centrale présente un problème: le miroir ne peut pas être courbé de façon brusque pour assurer ce saut de phase.

La transition nécessairement douce entre la zone centrale déphasée de $\pi$, et la zone latérale, se traduit par l'apparition d'un premier ordre quadratique à la fonction de phase, équivalent à un déplacement du foyer. La figure 4 présente la répartition d'intensité dans la zone focale, chaque « coupe » transverse étant normalisée, déterminée par un calcul de rétro-propagation sur la base des fronts d'ondes mesurés en sortie par le Shack-Hartmann. On constate l'apparition d'un décalage important entre la zone de minimum local d'intensité, et le foyer géométrique.

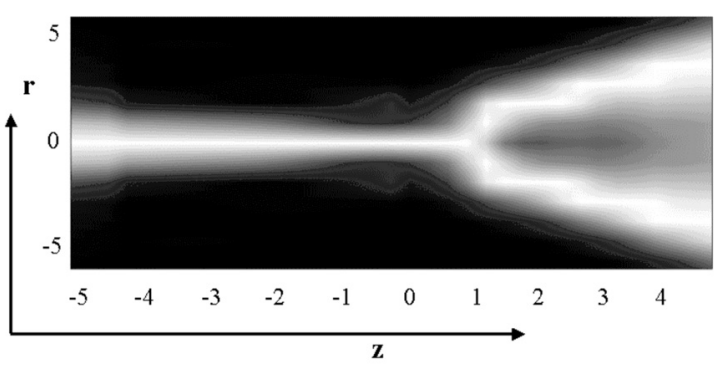

Figure 4. Propagation du faisceau avec le saut de phase suivant l'axe z. La répartition d'intensité est normalisée pour chaque $\mathrm{z}$.

On remarque que la profondeur de la zone où on observe le minimum local de l'intensité selon l'axe z, est égale à 3 longueurs de Rayleigh. Cette distance est beaucoup moins importante dans la méthode basée sur l'utilisation d'une lame demionde. Notre méthode présente donc l'avantage d'offrir un plus grand volume de piégeage pour l'accélération des électrons dans le faisceau laser. 


\section{PREMIÈRE APPLICATION DU CONTRÔLE SPATIAL AUX HARMONIQUES}

Pour observer les effets de la modification de l'intensité laser sur la génération d'harmonique nous avons placé la cellule de gaz dans la région d'interaction (figure 3). Le rayonnement harmonique est analysé par un spectromètre à réflexion et la caméra CCD. Nous avons commencé par corriger les aberrations du laser en sortie de chaîne. Nous avons ainsi obtenu une distribution d'intensité au foyer parfaitement gaussienne et atteint un éclairement dans le vide de $7.10^{15} \mathrm{~W} / \mathrm{cm}^{2}$. Le spectre harmonique obtenu dans ces conditions est montré sur la figure 5a. On observe six harmoniques consécutives (ordre 21-31) obtenues dans l'argon (pression 26 torr, longueur de la cellule $3 \mathrm{~mm}$ ).

a)

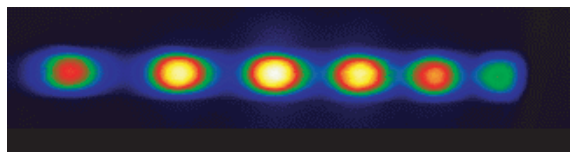

b)

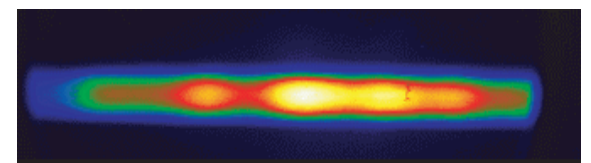

Figure 5. Spectres harmoniques enregistré par la CCD, avec un profil d'intensité Gaussien (a) et top-hat (b).

Le front d'onde est alors modifié pour donner au faisceau dans la zone d'interaction une forme spatiale proche d'un «top-hat», forme intermédiaire entre le minimum local (Figure $4, \mathrm{z}<-2 \mathrm{~mm}$ ) et un maximum central (figure $4, \mathrm{z}>-1 \mathrm{~mm}$ ). On observe alors un élargissement majeur de chaque harmonique, donnant un spectre s'apparentant à un quasi continuum (Figure 5b). Il est maintenant admis [9] que la génération d'harmoniques d'ordre élevé se traduit par l'existence d'un train d'impulsions attosecondes ; le plateau d'harmoniques devient un continuum lorsque l'émission est restreinte en temps à une «porte temporelle » de l'ordre d'une femtoseconde ; un flash unique est alors obtenu. Différentes propositions sont avancées aujourd'hui pour la mise en œuvre d'une telle porte [10]. Le spectre mesuré ici peut s'expliquer par l'apparition d'impulsions attosecondes uniques, dans une porte temporelle étroite due à un accord de phase très transitoire. D'après Kazamias et Balcou, une distribution d'intensité « top-hat » doit permettre de synchroniser les portes temporelles correspondant aux différents points du milieu d'interaction [11]. Des simulations complètes sont en cours, pour explorer en détails ce mécanisme de contrôle temporel attoseconde des harmoniques élevées via un contrôle spatial du mode laser dans le milieu.

En conclusion, nous avons présenté des résultats de contrôle actif des harmoniques d'ordre élevé, via des modifications de phases spectrales ou spatiales du laser. Le contrôle de phase spectrale permet en particulier d'obtenir une accordabilité presque complète des harmoniques élevées dans la gamme 20-40 nm ; le contrôle de phase spatiale fait apparaître des effets spectraux surprenants, compatibles avec l'obtention de portes temporelles d'accord de phase.

\section{Bibliographie}

[1] Brabec T. et Krausz F., Rev. Mod. Phys. 72 (2000) 545-591

[2] Tournois P., Opt. Commun. 140 (1997) 245-249

[3] Verluise P., Laude V., Cheng Z., Spielmann Ch. et Tournois P., Optics Letters 25 (2000) 575-577

[4] Verluise P., Laude V., Huignard J.-P., Tournois P., Migus A., J. Opt. Soc. Am. B 17 (2000) 138-145

[5] Chang Z., Phys. Rev. A Rapid Communications 58 (1998) R30-33

[6] Kazamias S. et al. Eur. Phys. Journal D 21 (2002) 353-359

[7] Reitze D. et al. Optics Letters 29 (2004) 86-88

[8] Chaloupka J.L. et al. J. Opt. Soc. Am. B 17 (2000) 5 713-722

[9] Paul P.M. et al. Science 292, (2001), 1689

[10] Tcherbakoff O. et al. Phys. Rev. A 68 (2003) 043804

[11] Kazamias S. et Balcou Ph., Phys. Rev. A 69 (2004) 063416-063421 